\title{
Using Points and Surfaces to Improve Voxel-Based Non-rigid Registration
}

\author{
T. Hartkens, D.L.G. Hill, A.D. Castellano-Smith, D.J. Hawkes ${ }^{1}$, \\ C.R. Maurer Jr. ${ }^{2}$, A.J. Martin ${ }^{3}$, W.A. Hall, H. Liu, and C.L. Truwit ${ }^{4}$ \\ 1 Computational Imaging Sciences Group, Guy's Hospital, King's College London,UK \\ 2 Department of Neurosurgery, Stanford University, Stanford, CA \\ ${ }^{3}$ Department of Radiology, University of California San Francisco, CA \\ 4 Departments of Radiology and Surgery, University of Minnesota, Minneappolis, MN
}

\begin{abstract}
Voxel-based non-rigid registration algorithms have been successfully applied to a wide range of image types. However, in some cases the registration of quite different images, e.g. pre- and post-resection images, can fail because of a lack of voxel intensity correspondences. One solution is to introduce feature information into the voxel-based registration algorithms in order to incorporate higher level information about the expected deformation. We illustrate using one voxelbased registration algorithm that the incorporation of features yields considerable improvement of the registration results in such cases.
\end{abstract}

\section{Introduction}

Increasing number of studies focus on detecting temporal anatomical changes in the brain by non-rigidly registering tomographic images, e.g. [2][15][14]. The resulting deformation field is used to quantify the volume change or the displacement of the tissue. The applied registration algorithms can be divided into feature-based approaches which use point, curves, and surface information to drive the registration, or voxel-based approaches which operate directly on the image intensities and define voxel similarity measure to compare the images. While feature-based registration algorithms can reliably align anatomical boundaries and therefore can quantify the change of certain anatomical structure with high precision, voxel-based registration algorithms use the intensities throughout the whole images and therefore yield deformation values based on the image content also in regions where it is difficult to detect distinct features. We demonstrate in this paper that incorporating feature information in a voxel-based registration algorithm combines the advantages of both approaches.

The voxel-based non-rigid registration algorithm with which we have the greatest experience works well on a wide variety of data including pre-and post contrast MR mamograms [11], serial MR images of the brain [7], and pre-and post resection brain images [6]. However the algorithm sometimes fails, especially when there are large changes between the images. In particular, in a series of 24 pre- and post- resection MR images of the brain, we found that 3 clearly failed on visual inspection to align corresponding features.

The technique we propose enables the user to optionally provide additional constraints to improve algorithm accuracy using semi-automatic point and surface delineation. We envisage that this approach could be used either on images which are expected 
to cause the algorithm difficulty, or could be used in cases where visual inspection shows the voxel-driven algorithm to have failed. Anatomical 3D landmarks and partial brain surfaces, respectively, are semi-automatically determined in regions where large deformation occurred. In comparison to an automatic approach, a semi-automatic approach has the advantage that the user can control the results and can introduce higher order information about the expected deformation. The feature information is combined with a voxel-based non-rigid registration algorithm. The extended registration algorithm is applied on 3D image pairs with substantial differences and which are therefore difficult to register. We demonstrate on three example images that neither the purely voxel-based registration algorithm nor using feature information only yields satisfying results and that the combination of feature and voxel information improves the registration results considerably.

Previously, Johnson and Christensen [8] presented an intensity-based consistent thinplate spline algorithm for 2D images using manually detected corresponding point landmarks and image intensities. In [1] an intensity-based thin-plate spline algorithm is applied on 3D images matching curves which represent cortical sulci. Similar features are incorporated in a optical flow algorithm using squared differences in [5]. Another paper [12] introduces an attribute vector for each voxel which contains the intensity, edge type, and a set of geometric moment invariants. None of these papers presents results on images with substantial changes, for example images where the deformation is neither one-to-one nor continuous. Additionally, they consider squared differences of the voxel intensities as similarity measure, which implies the same modality for the reference and source image, whereas we use Normalized Mutual Information.

\section{Methology}

The non-rigid registration algorithm deforms a regular grid of control points in the reference image by moving the control points while tissue motion is described by free-form deformation using B-spline interpolation between the control points[11]. Normalised Mutual Information (NMI) is used as the voxel-based similarity measure $C_{N M I}$ [7], which does not restrict the images to be from the same modality. The purely voxel-based algorithm was previously evaluated for the registration of 3D breast MR images [3], and it has been shown that the displacement vectors determined in deformed brain MR image data correspond in general well with manually determined measurements [6]. However, the algorithm sometimes fails to align anatomical structures correctly due to a lack of voxel correspondence near the changes, for example the true deformation may be neither one-to-one nor continuous. Our new algorithm allows the user to overcome these difficulties by incorporating point or surface information in a semi automated way.

\subsection{Corresponding Point Landmarks}

As corresponding points we consider point landmarks in the images, i.e., prominent points, where the surface of anatomical structures is strongly curved, e.g. the tip of the frontal horn of the ventricular system within in the human brain. Usually, such 3D landmarks are selected manually - a task which is tedious, time-consuming, and often 

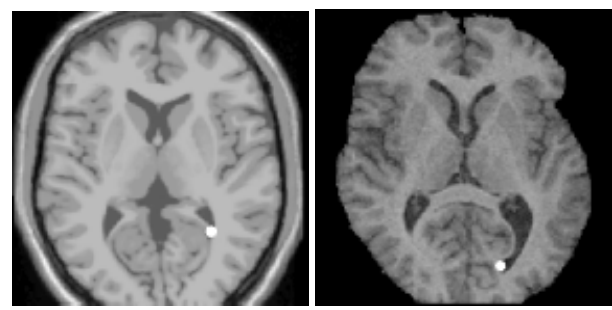

(a)
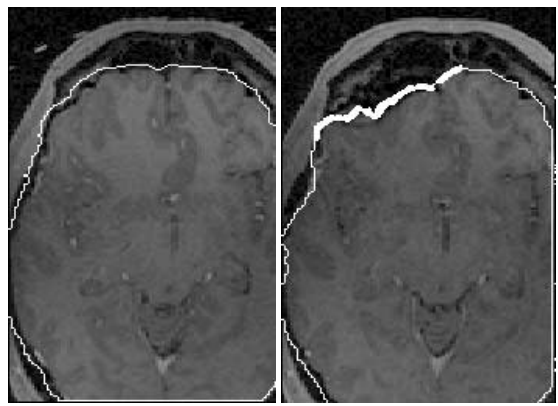

(b)

Fig. 1. (a) shows one corresponding point pair in the MNI atlas (left) and in the subject image (right). Altogether six 3D landmarks were detected in each image semi-automatically using the 3D operator (not displayed because they are in other slices). (b) shows the pre (left) and post (right) interventional MR images overlaid with a contour illustrating the segmentation obtained from the Brain Extraction Tool [13]. Only a part of the 3D surface of the post interventional image (reference image) is considered by the registration process (displayed as bold contour in the right image). This partial surface is determined manually via a GUI and extracted in a region where the displacement is substantial

lacks accuracy. The alternative we follow is a semi-automatic procedure for landmark extraction [9] which has the advantage that the user can interactively control the results. First, a coarse position of a certain landmark is determined manually. Second, a 3D operator is applied within a volume-of-interest (VOI) centred at the coarse position to detect potential landmark candidates. Third, the user selects the most promising candidate.

The 3D operator used in our investigation is based on the matrix $\underline{C}=\overline{\nabla g(\nabla g)^{T}}$ with the gradient $\nabla g=\left(g_{x}, g_{y}, g_{z}\right)^{T}$ of the image intensity function $g$ and is defined as [10]:

$$
k=\frac{\operatorname{det}(\underline{C})}{\operatorname{trace}(\underline{C})} .
$$

The extrema of the operator responses are considered as detections of the operator. In [4] it has been shown that this operator can reliably detect features including the tips of the lateral ventricle. The negative mean distance of the corresponding landmarks is taken as similarity measure

$$
C_{F}=-\frac{1}{n} \sum_{i}^{n}\left\|\boldsymbol{p}_{i}^{r}-\boldsymbol{p}_{i}^{s}\right\|
$$

where $\left(\boldsymbol{p}_{i}^{r}, \boldsymbol{p}_{i}^{s}\right)$ is the $i$-th corresponding point pair in the reference and source image.

\subsection{Surfaces}

In cases with substantial brain shift a partial brain surface is used to introduce pre-known displacement information. First, the brain was segmented using the publicly available 
Brain Extraction Tool (http://www.fmrib.ox.ac.uk/fsl) [13] and the marching cubes algorithm was applied on this segmentation to obtain a 3D brain surface representation. Second, a region of interest from this surface was extracted manually in the reference image (see Fig 1) supported by a 3D graphical user interface. The selected surface might, for example, be in the region of the resection where sub-dural air is present. Based on randomly chosen points $p_{i}^{r}$ on this partial surface, their closest points $p_{i}^{s}$ on the source surface are determined and established as corresponding point pairs $\left(\boldsymbol{p}_{i}^{r}, \boldsymbol{p}_{i}^{s}\right)$. The similarity of these point pairs is calculated as described in the previous section for the point landmarks. In contrast to the point landmarks these point pairs are not fixed during the registration process but in every iteration newly established. This allows the surfaces to slide over each other without changing the similarity measure, analogous to the way that the iterative closet point (ICP) algorithm works.

\subsection{Combining Voxel and Feature Similarity Measure}

The voxel similarity measure $C_{N M I}$ is linearly combined with the feature similarity measure $C_{F}$ :

$$
C_{\text {total }}=C_{N M I}+\tau \cdot C_{F}
$$

The optimisation process determines in each step the gradient of the similarity $C_{\text {total }}$ in relation to the degrees of freedom $d_{j}$, i.e. the displacement of the control points,

$$
\nabla_{d_{j}} C_{\text {total }}=\nabla_{d_{j}} C_{N M I}+\tau \cdot \nabla_{d_{j}} C_{F}
$$

and maximises the similarity along this gradient. Typically, the absolute value of the voxel similarity gradient $\nabla_{d_{j}} C_{N M I}$ has a different order of magnitude than the absolute value of the feature similarity gradient $\nabla_{d_{j}} C_{F}$. Therefore we weight the feature similarity gradients with the factor $\tau$ to scale it to the same order of magnitude. The parameter $\tau$ is calculated automatically once at the beginning of the registration process and estimated as the ratio of the similarity derivatives in the direction of their gradients:

$$
\tau=\frac{\frac{\partial C_{N M I}}{\partial \nabla C_{N M I}}}{\frac{\partial C_{F}}{\partial \nabla C_{F}}}
$$

\subsection{Image Data}

Our new algorithm was applied to images that had proven difficult to register in previous studies. The first image was one of a series of 11 subjects in a serial MR study that had involved non-rigid registration of the MNI atlas to each subject [7](Fig. 11) for the purpose of segmenting the lateral ventricle. Visual inspection had shown good registration for the other 10 subjects, but clear mis-registration in the vicinity of the lateral ventricles in this subject, who had an abnormal ventricular shape. We registered this subject using our new technique by semi-automatically detecting the tips of the frontal, occipital and temporal horns of both lateral ventricles. The second and third images we registered were selected from group of 24 patients imaged at the start and end of a MR guided neurosurgical intervention. Two images for which the purely voxel-based algorithm failed were chosen to apply the new approach (assigned with resection 8 and resection3, Fig. 2). 


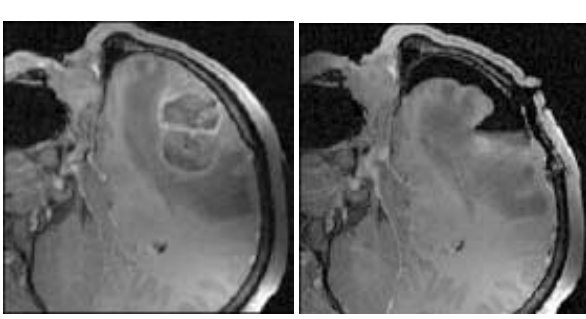

resection 8

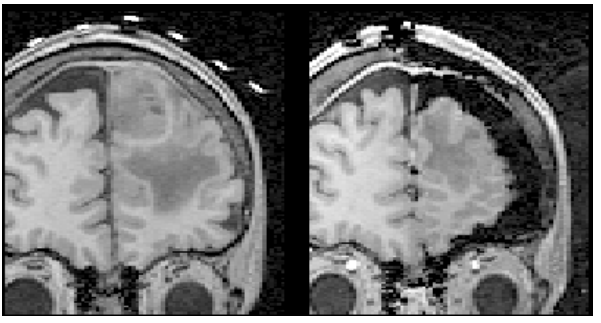

resection 3

Fig. 2. Pre- and post-procedure images

(a)
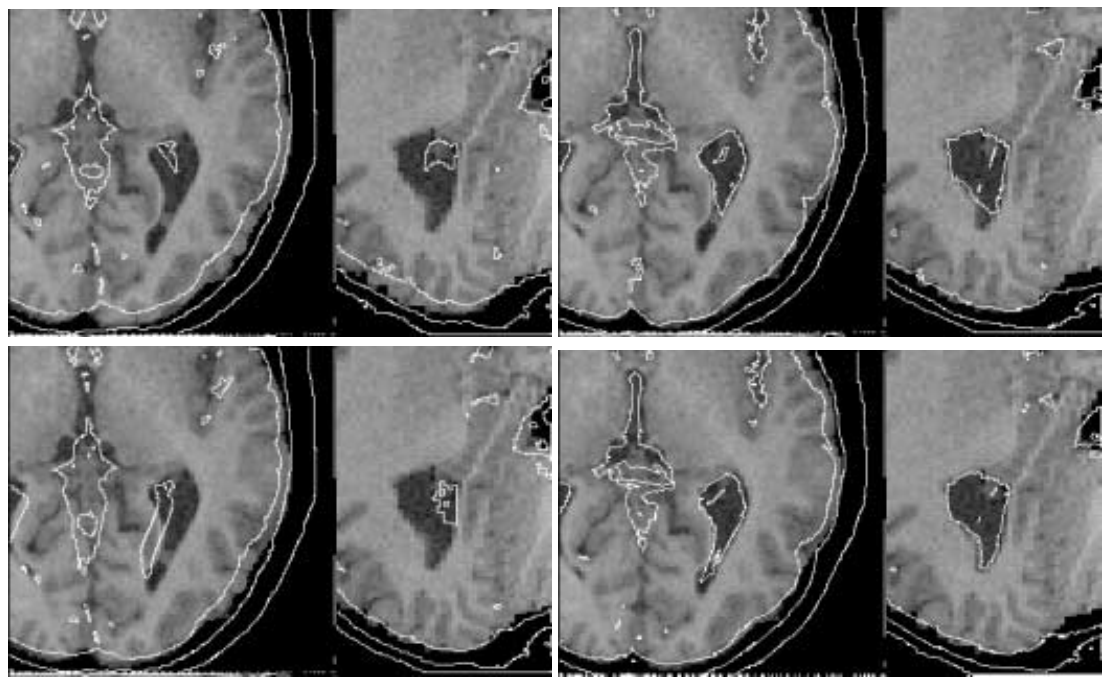

(b)

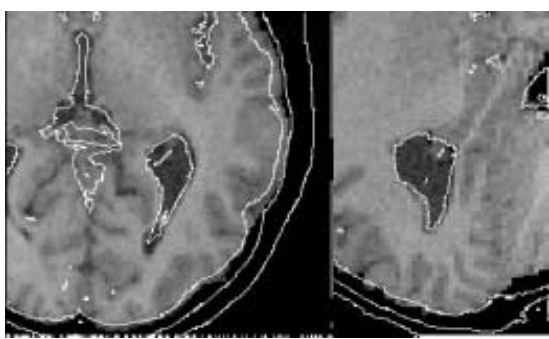

Fig. 3. Subject overlaid with the contour of the registered MNI atlas. (a) rigid registration, (b) non-rigid registration using only voxel similarity, (c) approximating the FFD using only feature information, (d) non-rigid registration using both voxel similarity and feature information

\section{Results}

In each Fig. 3, 4 and 5 the results after rigid-registration (a), after non-rigid registration using only voxel similarity (b), after approximating the FFD using only surface information (c), and after non-rigid registration using both voxel similarity and surface information (d) are shown for the investigated images.

Fig[3(d) shows that the boundary of the ventricle in the registered MNI atlas is well aligned with the ventricle of the subject image using voxel-intensities and point landmark information. The purely voxel-based algorithm 3 (b) yields reasonable results, but does not match the tip of the ventricle as well as the extended registration algorithm. The subtraction image in Fig 4 (b) after non-rigid registration without surface information demonstrates that the algorithm aligns the ventricular system and other structures inside the brain well, but does not detect the brain shift at the surface. In contrast, approximating 
(a)
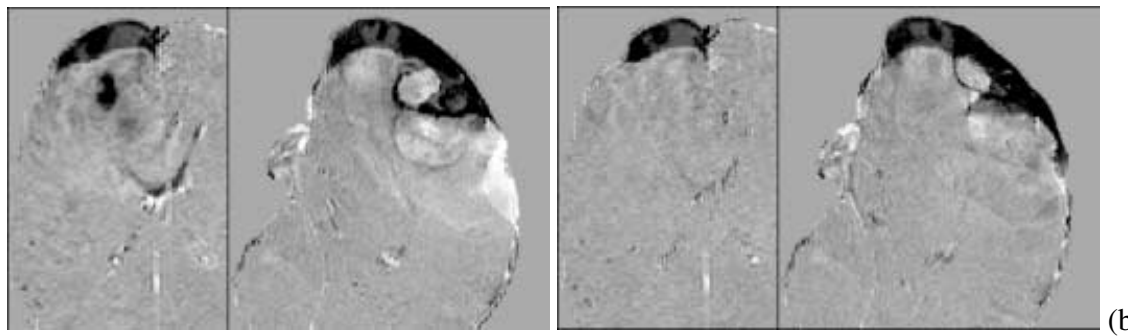

(c)
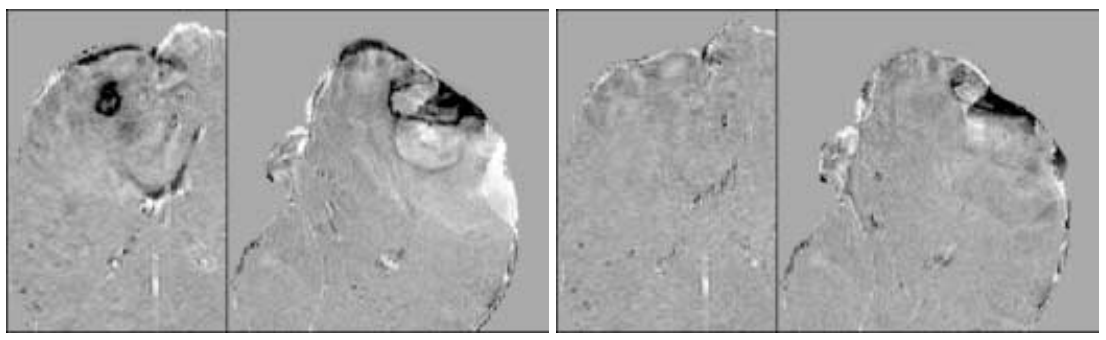

(b)

Fig. 4. Subtraction of post- and registered pre-resection image (resection8); see description for Fig 3

the FFD grid corresponding to the surface displacement 4(c) aligns the brain surfaces well, but does not improve the results deeper in the brain in comparison to the rigid registration because of the local support of the B-splines. Introducing surface information in the registration algorithm improves the results close to the brain surface and inside the brain 4(d). The pre-procedure brain surface in case resection3 (Fig.5] is poorly aligned with the post-procedure brain surface by the non-rigid registration only 5(b). The approximated FFD yields a clear displacement of the surfaces in the axial slice 5](c). The combination of voxel-intensities and feature information 5(d) aligns the surface considerablely better than the voxel based algorithm.

\section{Conclusion}

Previous studies successfully register tomographic images for a wide range of applications and modalities using purely voxel-based non-rigid registration algorithms. For instance, in [6] a voxel-based algorithm registers reliablely non-rigidly interventional MR images even with considerable changes. However, in some cases the registration of quite different images can fail because of a lack of voxel intensity correspondences. In this work, we demonstrate that introducing feature information in a voxel-based algorithm using Normalised Mutual Information as a similarity measure can improve the registration results considerably in such cases. Either point landmarks or a partial brain surfaces were detected semi-automatically in the reference and source image supported by a 3D graphical user interface. The mean distance of these features was taken as a similarity measure and was linearly combined with the voxel similarity measure introducing the parameter $\tau$. In contrast to previous approaches [8 1.5] which specify similar parameter manually, we automatically determine the parameter on the basis of the im- 
(a)
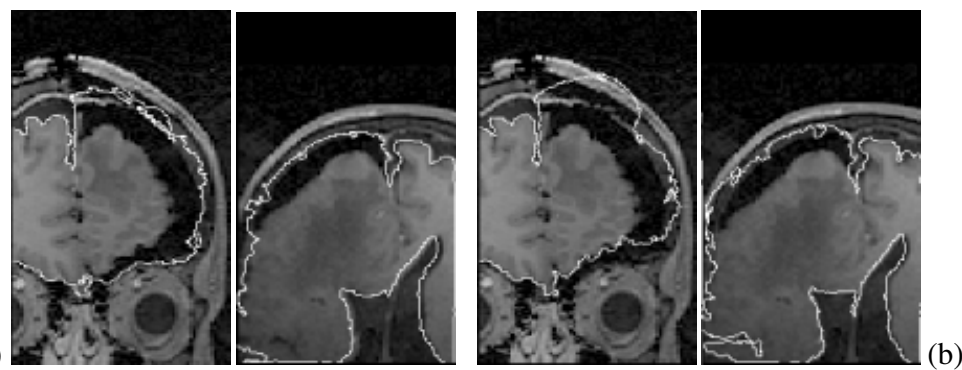

(c)
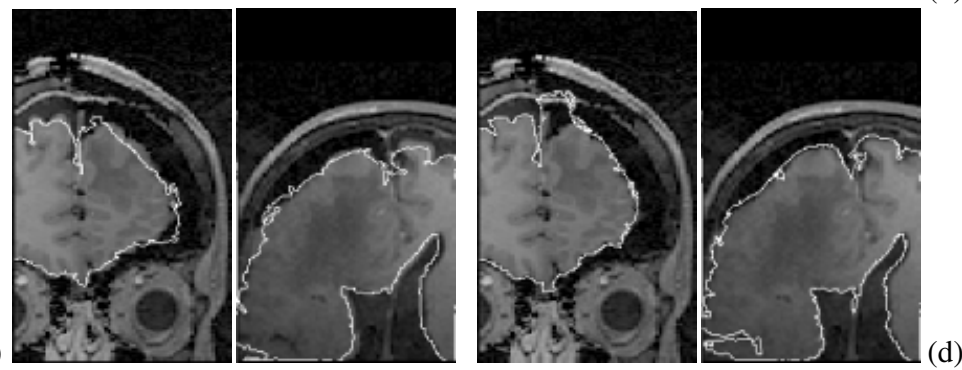

Fig. 5. Post-procedure image overlaid with the contour of the registered pre-procedure image (resection3); see description for Fig 3

age data. Additionally, our approach dynamically establishes point correspondence on the surface, permitting the surfaces to slide over each other. This might prove to be an advantage in cases where fixed point correspondences cannot be detected reliably in the images. Furthermore, the proposed semi-automatic procedure has the benefit that the user has the possibility to control the results and to introduce prior-knowledge about the expected transformation.

The extended registration algorithm has been applied on three 3D images with substantial differences. It turned out that using point and surface feature in the voxel-based registration algorithm aligns the boundary of the interested structure considerably better than a purely voxel-based algorithm. Since the results in other regions remain as good as the purely voxel-based algorithm, this initial study suggests that such an approach can combine the advantages of voxel-based and feature-based registration algorithms.

\section{References}

1. Pascal Cachier, Jean-Francois Mangin, Xavier Pennec, Denis Riviere, Dimitri PapadopoulosOrfanos, Jean Regis, and Nicholas Ayache. Multisubject non-rigid registration of brain mri using intensity and geometric features. In Niessen W and M. Viergever, editors, MICCAI2001, number 2208 in LNCS, pages 734-742. Springer-Verlag, 2001.

2. William R. Crum, A. Freeborough, and Nick C. Fox. The use of regional fast fluid registration of serial MRI to quantify local change in neurodegenerative disease. In D. Hawkes, D. Hill, and R. Gaston, editors, MICCAI99, pages 25-28, Oxford, July 1999. Springer-Verlag.

3. E. R. E. Denton, L. I. Sonoda, D. Rueckert, S. C. Rankin, C. Hayes, M. Leach, D. L. G. Hill, and D. J. Hawkes. Comparison and evaluation of rigid and non-rigid registration of breast $\mathrm{mr}$ images. JCAT, 23:800-805, 1999. 
4. T. Hartkens, K. Rohr, and H. S. Stiehl. Performance of 3D differential operators for the detection of anatomical point landmarks in MR and CT images. In Proc. SPIE's International Symposium Medical Imaging, Image Processing, pages 32-43, San Diego,CA,USA, Feb 1998.

5. Pierre Hellier and Christian Barillot. Cooperation between local and global approaches to register brain images. In R.M. Leahy MF. Insana, editor, IPMI2001, number 2082 in LNCS, pages 315-328. Springer-Verlag, 2001.

6. Derek L.G. Hill, Calvin R. Maurer, Alastair J. Martin, Saras Sabanathan, Walter A. Hall, David J. Hawkes, Daniel Rueckert, and Charles L. Truwit. Assessment of intraoperative brain deformation using interventional MR imaging. In Chis Taylor and Alan Colchester, editors, MICCAI'99, Lecture Notes in Computer Science 1679, pages 910-919, Cambridge, UK, September 1999. Springer-Verlag.

7. Mark Holden, Derek L.G. Hill, Erika R.E. Denton, Jo M. Jarosz, Tim C.S. Cox, Torsten Rohlginf, Joanne Goodey, and David J. Hawkes. Voxel similarity measures for 3D serial mr brain image registration. IEEE Transactions on Medical Imaging, 19(2):94-102, Feb 2000.

8. Hans J. Johnson and Gary E. Christensen. Landmark and intensity-based, consistent thinplate spline image registration. In R.M. Leahy M.F. Insana, editor, IPMI2001, number 2082 in LNCS, pages 329-343. Springer-Verlag, 2001.

9. K. Rohr, H.S. Stiehl, R. Sprengel, T.M. Buzug, J. Weese, and M.H. Kuhn. Landmark-based elastic registration using approximating thin-plate splines. IEEE Trans. on Medical Imaging, 20(6):526-534, June 2001.

10. K. Rohr. On 3D differential operators for detecting point landmarks. Image and Vision Computing, 15(3):219-233, March 1997.

11. D. Rueckert, L. I. Sonoda, C. Hayes, D. L. G. Hill, M.O. Leach, and D. J. Hawkes. Non-rigid registration using free-form deformations: Application to breast MR images. IEEE Trans. Medical Imaging, 18(8):712-721, 1999.

12. Dinggang Shen and Christos Davatzikos. Hammer: Hierarchical attribute matching mechanism for elastic registration. In Lawrence Staib, editor, IEEE Workshop on Mathematical Methods in Biomedical Image Analysis (MMBIA), pages 32-39. IEEE Computer Society, 2001.

13. S.M. Smith. Robust automated brain extraction. In Sixth Int. Conf. on Functional Mapping of the Human Brain, page 625, 2000.

14. C. Studholme, E. Novotny, I.G. Zubal, and J.S. Duncan. Estimating tissue deformation between functional images induced by intracranial electrode implantation using anatomical mri. NeuroImage, 13:561-576, 2001.

15. Paul M. Thompson, Jay N. Giedd, Roger P. Woods, David MacDonald, Alan C.Evans, and Arthur W. Toga. Growth patterns in the developing brain detected by using continuum mechanical tensor maps. Nature, 404(9):190-193, March 2000. 